\title{
ESTUDO COMPARATIVO, PROSPECTIVO E RANDOMIZADO ENTRE DOIS MÉTODOS DE ANESTESIA PARA CIRURGIA DO OMBRO
}

\author{
PROSPECTIVE RANDOMIZED STUDY COMPARING TWO ANESTHETIC \\ METHODS FOR SHOULDER SURGERY
}

Roberto Yukio Ikemoto', Joel Murachovsky², Luis Gustavo Prata Nascimento ${ }^{3}$, Rogerio Serpone Bueno4, Luiz Henrique Oliveira Almeida ${ }^{4}$, Eric Strose ${ }^{3}$, Sérgio Cabral de Mello ${ }^{5}$, Deise Saletti ${ }^{6}$

\section{RESUMO}

Objetivo: Avaliar a eficácia do bloqueio do nervo supraescapular associado à infusão de anestésico no espaço subacromial e compará-lo ao bloqueio interescalênico. Método: Avaliamos, prospectivamente e comparativamente, a eficácia dos métodos anestésicos em 45 pacientes com lesões pequenas ou médias isoladas do tendão do supraespinhal submetidos ao reparo artroscópico. Os pacientes foram divididos aleatoriamente em três grupos de 15, cada um com uma diferente combinação de métodos anestésicos. A eficácia da analgesia pós-operatória foi mensurada utilizando-se a escala visual da dor e consumo de drogas analgésicas, anti-inflamatórias e opioides. O consumo de anestésicos inalatórios durante a cirurgia também foi comparado entre os grupos. Resultados: A análise estatística não encontrou diferenças estatisticamente significativas entre os grupos com relação ao consumo de anestésico durante a cirurgia e a eficácia analgésica pós-operatória nas primeiras 48 horas. Conclusão: O bloqueio do nervo supraescapular associado à infusão de anestésico no espaço subacromial é uma excelente alternativa ao bloqueio interescalênico, particularmente em hospitais que não dispõem do estimulador elétrico de nervo.

Descritores - Bainha rotadora; Ombro; Artroscopia; Anestesia; Bloqueio nervoso

\section{ABSTRACT}

Objective: To evaluate the efficacy of suprascapular nerve block in combination with the infusion of anesthetic into the subacromial space compared to an interscalene block. Methods: Forty-five patients with small or medium isolated supraspinatus tendon lesions who underwent arthroscopic repair were prospectively evaluated and randomly assigned to three groups of 15 , each with a different combination of anesthetic methods. The efficacy of post-surgical analgesia was measured using the visual analogue scale for pain, and analgesic, anti-inflammatory, and opioid drug consumption. Inhalation anesthetic consumption during surgery was compared among the groups. Results: Statistical analysis found no significant differences among the groups regarding anesthetic consumption during surgery or postoperative analgesic efficacy during the first 48 hours. Conclusion: Suprascapular nerve block with infusion anesthetic into the subacromial space is an excellent alternative to interscalene block, particularly in hospitals where a nerve stimulator device is unavailable.

Keywords - Rotator cuff; Shoulder; Arthroscopy; Anesthesia; Nerve block

\footnotetext{
1 - Mestre em Medicina e Chefe do Grupo de Cirurgia do Ombro e Cotovelo da Faculdade de Medicina do ABC (FMABC).

2 - Doutor em Medicina e Assistente do Grupo de Cirurgia do Ombro e Cotovelo, FMABC.

3 - Mestre em Medicina e Assistente do Grupo de Cirurgia do Ombro e Cotovelo, FMABC.

4 - Pós-Graduando em Ciências da Saúde e Assistente do Grupo de Cirurgia do Ombro e Cotovelo, FMABC.

5 - Estagiário do Grupo de Cirurgia do Ombro e Cotovelo, FMABC.

6 - Anestesiologista do Hospital Estadual Mário Covas, FMABC.
}

Trabalho realizado na Disciplina de Doenças do Aparelho Locomotor da Faculdade de Medicina do ABC e no Hospital Estadual Mário Covas. Correspondência: Rua Monte Alegre, 253, 121 - 09531-110 - São Caetano do Sul (SP) - Brasil. E-mail: rob.ike@hotmail.com 


\section{INTRODUÇÃO}

Após um procedimento cirúrgico na articulação do ombro, muitos pacientes relatam dor intensa, especialmente durante as primeiras 48 horas, o que faz com que a maioria utilize grandes quantidades de analgésicos durante esse período ${ }^{(1,2)}$.

Com o advento da cirurgia artroscópica, muitas dessas cirurgias passaram a se tornar procedimentos ambulatoriais. No entanto, a dor ainda preocupa médicos e pacientes, uma vez que o seu controle fora do ambiente hospitalar, usando analgésicos endovenosos, torna-se impraticável ${ }^{(3)}$.

Na maioria das instituições, a artroscopia de ombro é realizada sob anestesia geral associada ao bloqueio interescalênico (BIE) ${ }^{(4)}$. Contudo, são descritas algumas complicações como intoxicação cardíaca, perda da consciência pela administração do anestésico e pneumotórax ${ }^{(5)}$. Além disso, sua eficácia depende da experiência do anestesiologista, uma vez que, em muitos dos nossos hospitais, o estimulador elétrico de nervo não está disponível ${ }^{(6)}$.

Recentemente, novas técnicas de anestesia vêm sendo estudadas, com avaliação de sua eficácia no intra e no pós-operatório, como a infusão contínua de ropivacaína no espaço subacromial; a anestesia intra-articular e o bloqueio do nervo supraescapular (BNSE) ${ }^{(2,7,8)}$.

O objetivo deste estudo foi o de avaliar, de forma prospectiva, a eficácia intra e pós-operatória do BNSE associado à infusão de anestésico no espaço subacromial e compará-lo ao BIE sem o uso do estimulador elétrico de nervo.

\section{MATERIAIS E MÉTODOS}

Entre janeiro de 2006 e novembro de 2007, todos os pacientes com lesões do manguito rotador foram candidatos para o estudo. Incluímos no trabalho 45 pacientes com lesões pequenas ou médias (de um total de 157 artroscopias realizadas no serviço para reparo de lesão do manguito rotador nesse período), classificadas pela ressonância magnética, com indicação de reparo artroscópico e sem história de cirurgia prévia no ombro acometido. Pacientes com lesões grandes ou extensas foram excluídos, assim como aqueles que não concordaram em participar do estudo. Os pacientes foram selecionados dentre aqueles que foram encaminhados ao Grupo de Cirurgia de Ombro e Cotovelo do Hospital Estadual Mário Covas, em Santo André, pelos médicos da assistência primária da região do ABC.
Os pacientes foram divididos aleatoriamente em três grupos diferentes. A randomização foi realizada pelo método de minimização, assegurando que os três grupos apresentassem número similar de pacientes. Os pacientes do grupo A foram submetidos ao reparo artroscópico da lesão do manguito rotador sob anestesia geral associada com BIE sem uso de estimulador elétrico de nervo. Os pacientes do grupo B foram submetidos à anestesia geral associada ao BNSE e infusão de anestésico no espaço subacromial momentos antes do procedimento cirúrgico, e os pacientes do grupo $\mathrm{C}$ foram submetidos ao mesmo procedimento artroscópico, porém apenas sob anestesia geral, e somente após o procedimento cirúrgico foi realizado o BNSE e a infusão do anestésico no espaço subacromial, para avaliar o consumo de anestésico durante o procedimento cirúrgico como grupo controle. Todos os procedimentos anestésicos foram realizados por apenas um anestesiologista experiente do hospital.

Dos 45 pacientes, 32 eram do sexo feminino e 13 do masculino. Não foi excluído nenhum paciente durante o período avaliado. A idade variou de 39-76 anos (média de 56 anos), e o lado dominante foi acometido em 33 pacientes.

No grupo A, 10 pacientes eram do sexo feminino e cinco do masculino. A média de idade foi de 54 anos (variando de 39 a 65 anos). O ombro dominante foi acometido em 11 casos. No grupo B, 11 pacientes eram do sexo feminino e quatro do masculino. A média de idade foi de 57 anos (variando de 45 a 69 anos) e o lado dominante acometido em 12 pacientes. No grupo C, 11 pacientes eram do sexo feminino e quatro do masculino. A média de idade foi de 57 anos (variando de 47 a 76 anos) e o ombro dominante foi acometido em 10 pacientes.

Em todos os pacientes a medicação pré-anestésica utilizada foi midazolam (3mg), cefalotina (2g) e ranitidina (50mg). A indução da anestesia geral foi realizada com alfentanyl (40mg/kg); propofol (3mg/kg) e rocuronium $(0,6 \mathrm{mg} / \mathrm{kg})$ e a manutenção realizada com 3l/ min de $\mathrm{O}_{2}$ a $50 \% / \mathrm{NO}_{2}$ mais $2 \%$ de isofluorano. O BIE foi realizado com $2 \mathrm{mg} / \mathrm{kg}$ de ropivacaína a $0,5 \%$ e o BNSE utilizou-se $2 / 3$ de $2 \mathrm{mg} / \mathrm{kg}$ de ropivacaína a 0,5\%. O terço restante foi aplicado no espaço subacromial. Em todos os casos a anestesia foi revertida com atropina $(0,01 \mathrm{mg} / \mathrm{kg})$ e prostigmina $(0,02 \mathrm{mg} / \mathrm{kg})$.

Calculamos o consumo de anestésico inalatório durante o procedimento cirúrgico a fim de compará-lo entre os grupos. 
Os procedimentos artroscópicos foram realizados, reparando a lesão do tendão do supraespinhal com âncoras de sutura, assim como, realizando acromioplastia.

Após a cirurgia todos os pacientes foram imobilizados com tipoia funcional e receberam analgésicos simples, analgésicos opioides e anti-inflamatório, aplicados conforme a solicitação do paciente. As quantidades de droga utilizada em cada grupo, nas primeiras 48 horas, foram comparadas entre os três grupos.

Para avaliação da eficácia anestésica de cada grupo, foi realizada a comparação da intensidade de dor durante o período pós-operatório de cada paciente por meio da escala visual da dor (VAS) (Figura 1) ${ }^{(9)}$. Todos foram questionados por um médico que desconhecia qual o método anestésico que o paciente havia sido submetido. A avaliação foi realizada tanto no período pré-operatório, como também, no pós-operatório imediato e nas primeiras oito, 16, 24 e 48 horas após a cirurgia. Outro parâmetro estudado foi o consumo das drogas analgésicas, anti-inflamatórias e opioides durante as primeiras 48 horas.

A análise estatística foi realizada utilizando-se o software SPSS (Statistical Package for Social Sciences) versão 13.0, para comparar os três grupos. Para a análise das variáveis paramétricas (idade, peso, escore de dor, consumo de anestésico e analgésicos) e das não paramétricas (sexo e lado acometido) foram utilizados os testes de Kruskal-Wallis e Mann-Whitney com significância quando $\mathrm{P}<0,05$.

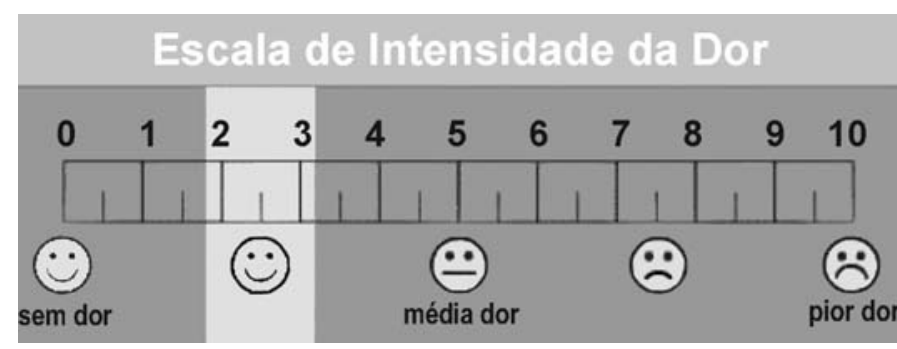

Figura 1 - Escala visual da dor (VAS)

\section{RESULTADOS}

Durante o procedimento cirúrgico, o consumo médio de anestésico inalatório (isofluorano) no grupo A foi de 32,2ml (variando de 9 a 65ml), no grupo B foi de $40 \mathrm{ml}$ (variando de 20 a $65 \mathrm{ml}$ ) e no grupo $\mathrm{C}$ foi de $43,6 \mathrm{ml}$ (variando de 25 a 80ml) (Figura 2).

No pré-operatório, a intensidade média de dor no grupo A foi de 7,6 (variação: 5-10). Durante o pós-operatório imediato, a intensidade média de dor relatada foi

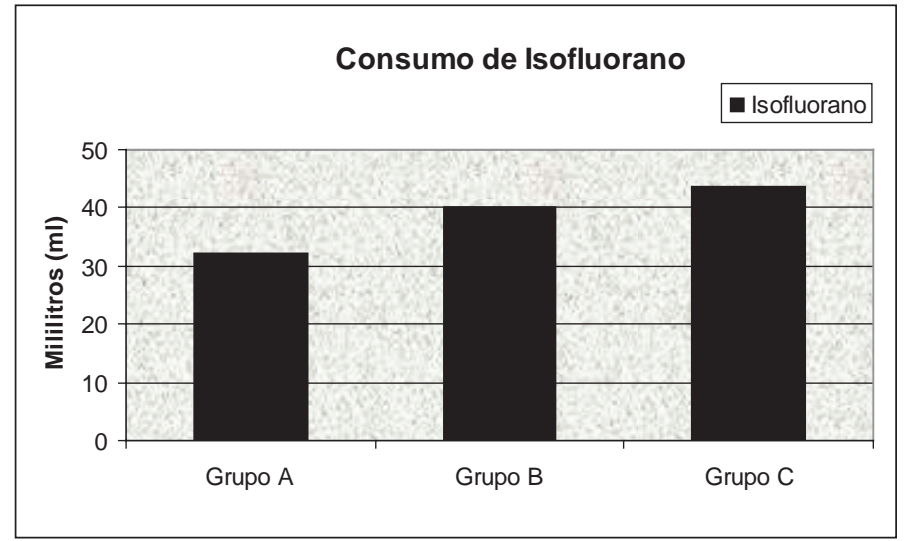

Fonte: SAME (Serviço de Arquivos Médicos do Hospital)

Figura 2 - Consumo médio de anestésicos inalatórios em mililitros (ml) nos grupos A, B e C

de 6,2 (variação: 0-10). Na avaliação pós-operatória de oito horas foi relatada média de 5,6 (variação: 0-10). Nas 16 horas do pós-operatório os pacientes relataram média da intensidade da dor de 4,5 (variação: 0-8), e nas 24 horas a média foi de 3,8 (variação: 0-9). Finalmente, nas 48 horas de pós-operatório foi relatada média da intensidade da dor de 3,0 (variação: 0-8).

No grupo $\mathrm{B}$, a intensidade média de dor no pré-operatório foi de 6,6 (variação: 5-9). Durante o pós-operatório imediato, a intensidade média de dor relatada foi de 5,8 (variação: 0-10) e na avaliação pós-operatório de oito horas foi relatada média de 5,5 (variação: 0-10). No pósoperatório de 16 horas os pacientes relataram média da intensidade da dor de 5,8 (variação: 2-10) e nas 24 horas a média foi de 5,1 (variação: 2-8). Finalmente, nas 48 horas de pós-operatório foi relatada média da intensidade da dor de 3,5 (variação: 0-5) (Tabela 1).

E, finalmente, no grupo $\mathrm{C}$, a intensidade média de dor no pré-operatório foi de 7,1 (variação: 0-10). Durante o pós-operatório imediato, a intensidade média de dor relatada foi de 6,0 (variação: 0-10) e no pós-operatório de

Tabela 1 - Média de pontuação da dor segundo a escala visual da dor (VAS) em cada grupo

\begin{tabular}{c|c|c|c}
\hline Momentos de Observação & Grupo A & Grupo B & Grupo C \\
\hline PRE_OP & 7,67 & 6,6 & 7,13 \\
\hline POI & 6,27 & 5,87 & 6,07 \\
\hline 8H_PO & 5,67 & 5,53 & 5,4 \\
\hline 16H_PO & 4,53 & 5,8 & 4,67 \\
\hline 24H_PO & 3,8 & 5,13 & 4,33 \\
\hline 48H_PO & 3 & 3,53 & 2,33 \\
\hline
\end{tabular}

Legenda: PRE_OP - pré-operatório, POI - pós-operatório imediato, H - Horas, PO - pósoperatório

Fonte: SAME (Serviço de Arquivos Médicos do Hospital) 
oito horas foi relatada média de 5,4 (variação: 0-10). No pós-operatório de 16 horas, os pacientes relataram média da intensidade da dor de 4,6 (variação: 0-7) e nas 24 horas a média foi de 4,3 (variação: 0-10). Finalmente, nas 48 horas de pós-operatório foi relatada média da intensidade da dor de 2,5 (variação: 0-6).

$\mathrm{Na}$ análise do consumo de drogas do grupo A, o consumo foi de 5,4 ampolas de analgésicos, 2,7 ampolas de anti-inflamatórios e 0,8 ampola de opioides. No grupo $\mathrm{B}$, o consumo foi de 3,1 ampolas de analgésicos, 1,05 ampolas de anti-inflamatórios e 1,2 ampolas de opioides. E, no grupo C, o consumo foi de 4,2 ampolas de analgésicos, 2,2 ampolas de anti-inflamatórios e uma ampola de morfina (Figura 3).

De acordo com a análise estatística, não foram encontradas diferenças estatisticamente significantes $(\mathrm{P}>0,05)$ em relação à amostra de pacientes (idade, peso, sexo e lado acometido) na comparação entre os três grupos. O consumo de anestésico inalatório durante a cirurgia não mostrou diferença estatisticamente significante entre os três grupos de acordo com o teste de Kruscall-Wallis ( $\mathrm{P}=0,216)$ (Figura 2), mas, em média, houve consumo de $7,8 \mathrm{ml}$ a menos no grupo A quando comparado ao grupo $\mathrm{B}$, e $11,4 \mathrm{ml}$ a menos quando comparado ao grupo C.

Com relação à avaliação da dor, os três grupos mostraram eficácia analgésica similar no período pósoperatório e não houve diferença estatística de acordo com o teste de Kruscall-Wallis em qualquer momento da interrogação sobre a intensidade da dor (período préoperatório $\mathrm{P}=0,140$; pós-operatório (pós-op) imediato: $\mathrm{P}=0,872$; oito horas pós-op: $\mathrm{P}=0,969 ; 16$ horas pósop: $\mathrm{P}=0,383$; 24 horas pós-op: $\mathrm{P}=0,203$; e 48 horas pós-op: $\mathrm{P}=0,260$ ) (Tabela 1 ).

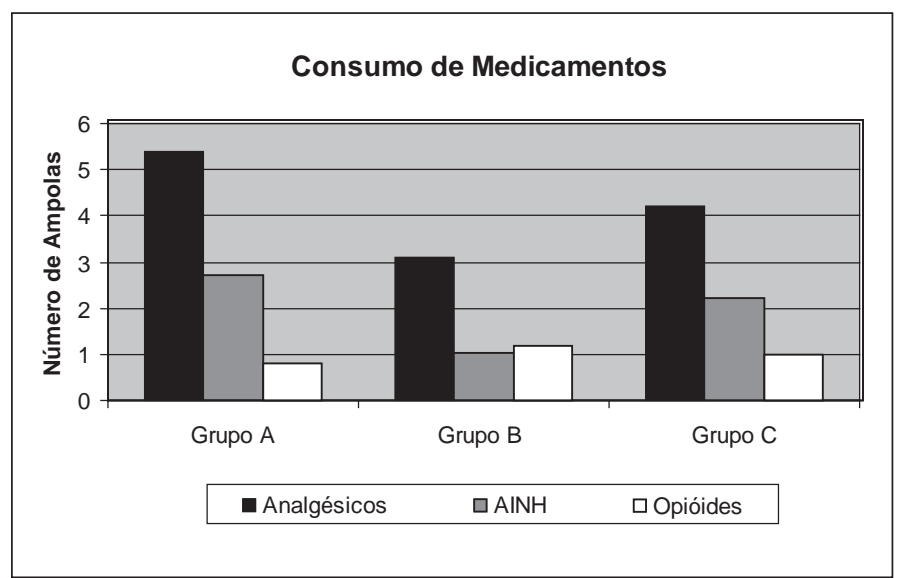

Legenda: AINH - Anti-inflamatório não hormonal

Fonte: SAME (Serviço de Arquivos Médicos do Hospital)

Figura 3 - Número médio de ampolas consumidas de analgésicos, anti-inflamatórios e opioides no pós-operatório em cada grupo
Em relação ao consumo de drogas analgésicas e antiinflamatórias, de acordo com o teste de Kruscall-Wallis, também não houve diferença estatisticamente significante entre os três grupos (analgésicos: $\mathrm{P}=0,073$; opioides: $\mathrm{P}=$ 0,88; e anti-inflamatórios: $\mathrm{P}=0,880$ ) (Figura 3), mostrando eficácia similar entre os tipos de anestesia empregados.

\section{DISCUSSÃO}

A anestesia geral associada ao BIE é amplamente usada como método anestésico nas cirurgias do ombro, incluindo as $\operatorname{artroscopias}^{(4,10)}$. No entanto, no Brasil e em muitos países em desenvolvimento, apenas alguns hospitais privados e uma minoria dos hospitais públicos dispõem do estimulador elétrico de nervo para a realização do bloqueio anestésico. O número de complicações pode aumentar quando esta técnica é realizada sem o uso do estimulador, como relatado por Weber e Jain ${ }^{(5)}$, que relatam intercorrências como episódios de colapso cardiovascular, grave depressão respiratória, alterações neurológicas persistentes por até seis semanas e dor persistente no período pósoperatório $^{(11)}$.

Laurila et al ${ }^{(12)}$ compararam o BIE à infiltração de $15 \mathrm{ml}$ de ropivacaína ( $5 \mathrm{mg} / \mathrm{ml})$ no espaço subacromial, imediatamente após a introdução do artroscópio no ombro. Relataram que esse método não reduziu a dor pós-operatória nem o consumo de oxicodeína quando comparado ao BIE.

Harvey et $a^{(6)}$ realizaram estudo duplo cego para avaliar a analgesia após a acromioplastia artroscópica. Eles compararam a infusão contínua de 0,2\% de ropivacaína no espaço subacromial à infusão de solução salina a 0,9\%, usado como grupo controle. Utilizando a escala visual da dor (VAS), notaram que o grupo em que foi utilizada a ropivacaína relatou 34\% menos dor pós-operatória (46\% no primeiro dia e $22 \%$ no segundo), com diferença estatística significante.

Em outro estudo, Muittari et al ${ }^{(13)}$ encontraram melhor analgesia em pacientes que, após procedimentos de acromioplastia aberta sob anestesia geral, recebiam $10 \mathrm{ml}$ de $0,5 \%$ de bupivacaína no espaço subacromial quando comparados àqueles que receberam $10 \mathrm{ml}$ de oxicodeína no espaço subacromial e $5 \mathrm{mg}$ intramuscular.

Almeida et al $^{(14)}$ analisaram três grupos de pacientes que foram submetidos ao reparo artroscópico do manguito rotador sob anestesia geral. Um dos grupos foi submetido ao BIE, o segundo grupo ao BIE associado a $150 \mathrm{mg}$ de clonidina e, o terceiro, submetido à infiltração dos portais com $10 \mathrm{ml}$ de ropivacaína e $20 \mathrm{ml}$ no espaço subacromial após a cirurgia, após a administração contínua de 0,2\% de ropivacaína. A avaliação da intensidade da dor com a 
VAS nas 24 horas do período pós-operatório mostrou que o terceiro grupo apresentava eficácia analgésica superior quando comparado aos outros grupos $(\mathrm{P}<0,001)$. Porém, o BIE foi realizado sem o estimulador elétrico.

Em estudo randomizado, duplo cego, Singelyn et al ${ }^{(15)}$ compararam a eficácia da anestesia intra-articular (AIA) ao BNSE e BIE com estimulador elétrico realizando acromioplastia artroscópica em 120 pacientes. Os autores encontraram que os grupos de BNSE e BIE apresentaram menos dor no pós-operatório quando comparados ao grupo da AIA, apesar de não haver diferença estatística. Também encontraram que o grupo do BNSE tinha o menor consumo de morfina e menos efeitos adversos e concluíram que o BIE é a técnica mais eficaz para a realização de acromioplastia artroscópica e que, quando o BIE for contraindicado, o BNSE é alternativa eficiente.

Quando comparamos nossos resultados com o estudo de Laurila et $a^{(12)}$ vemos que este também não notou diferença da analgesia do BIE e da infiltração de bupivacaína no espaço subacromial; entretanto, não foi realizado o BNSE. Já no estudo de Almeida et al ${ }^{(14)}$, encontrou-se maior eficácia analgésica com a infiltração de anestésico no espaço subacromial, diferindo de nossos resultados. Sendo que, também não realizaram o BNSE e, como em nosso trabalho, o BIE foi realizado sem estimulador elétrico. No estudo de Singelyn et $a^{(15)}$, assim como em nosso estudo, não houve diferença estatística entre os grupos, resultados similares aos encontramos na literatura.

Neste estudo preliminar, comparamos a eficácia analgésica entre a técnica do BIE usando $2 \mathrm{mg} / \mathrm{kg}$ de ropivacaína a 0,5\%, à técnica do BNSE usando 2/3 de 2mg/ $\mathrm{kg}$ de ropivacaína a $0,5 \%$ associado à infusão do $1 / 3$ restante no espaço subacromial momentos antes da ci-

\section{REFERÊNCIAS}

1. Boss AP, Maurer T, Seiler S, Aeschbach A, Hintermann B, Strebel S. Continuous subacromial infusion for postoperative analgesia after open acromioplasty and rotator cuff repair: preliminary results. J Shoulder Elbow Surg. 2004;13(6):630-4.

2. Barber FA. Suprascapular nerve block for shoulder arthroscopy. Arthroscopy. 2005;21(8):1015:e1-e4.

3. Scoggin JF, III, Mayfield G, Awaya DJ, Pi M, Prentiss J, Takahashi J. Subacromial and intra-articular morphine versus bupivacaine after shoulder arthroscopy. Arthroscopy. 2002;18(5):464-8.

4. Karkabi S, Besser M, Zinman C. Arthroscopic subacromial decompression performed under local anesthesia. Arthroscopy. 2005;21(11):1404:e1-1404e2.

5. Weber SC, Jain R. Scalene regional anesthesia for shoulder surgery in a community setting: an assessment of risk. J Bone Joint Surg Am. 2002;84(5):775-9.

6. Harvey GP, Chelly JE, AISamsam T, Coupe K. Patient-controlled ropivacaine analgesia after arthroscopic subacromial decompression. Arthroscopy. 2004;20(5):451-5.

7. Arciero RA, Taylor DC, Harrison SA, Snyder RJ, Leahy KE, Uhorchak JM. Interscalene anesthesia for shoulder arthroscopy in a community-sized military hospital. Arthroscopy. 1996;12(6):715-9.

8. Savoie FH, Field LD, Jenkins N, Mallon WJ, Phelps RA 2nd. The pain control infusion pump for postoperative pain control in shoulder surgery. rurgia e, à mesma técnica do BNSE imediatamente após o procedimento. Observamos eficácia analgésica similar entre as três técnicas empregadas, uma vez que não houve diferenças estatisticamente significantes entre os grupos quando analisamos o consumo de medicamentos e a avaliação da dor, mostrando nesta pequena amostra de 45 pacientes que o BNSE associado à infusão de anestésico no espaço subacromial é uma técnica eficaz.

Uma das falhas de nosso estudo pode ser o tamanho da amostra de pacientes em cada grupo, uma vez que não obtivemos diferença estatisticamente significante entre os grupos e não sabemos se com uma amostra maior poderíamos ter resultados diferentes. Entretanto, agora temos dados importantes que podem auxiliar no cálculo da amostra necessária para obter-se um estudo com maior poder estatístico, certamente com número maior de pacientes em cada grupo. Além disso, acreditamos que este estudo pôde mostrar, para aqueles hospitais em que o estimulador não estiver disponível, que o BNSE pode ser uma boa opção uma vez que pode reduz as chances de complicações e que sua eficácia analgésica no pósoperatório é semelhante ao BIE.

Por outro lado, como não utilizamos o estimulador elétrico, o índice de falha dos bloqueios podem ter sido mais elevado, com maior consumo médio de anestésico durante a cirurgia, assim como de analgésico no pósoperatório.

\section{CONCLUSÃO}

Observamos eficácia semelhante entre o bloqueio do nervo supraescapular associado à infusão de anestésico no espaço subacromial comparada com o bloqueio interescalênico do plexo braquial.

Arthroscopy. 2000;16(4):339-42.

9. Jensen MK, Karoly P. Self-report scales and procedures for accessing pain in adults. In: Turk DC, Melzack R, editors. Handbook of pain assessment. New York: Guilford Press; 1992. p. 135-51.

10. Horn EP, Schroeder F, Wilhelm S, Wappler F, Sessler DI, Uebe B, et al. Wound infiltration and drain lavage with ropivacaine after major shoulder surgery. Anesth Analg. 1999;89(6):1461-6.

11. Mallon WJ, Thomas CW. Patient-controlled lidocaine analgesia for acromioplasty surgery. J Shoulder Elbow Surg. 2000;9(2):85-8.

12. Laurila PA, Löppönen A, Kanga-Saarela T, Flinkkilä T, Salomäki TE. Interscalene brachial plexus block is superior to subacromial bursa block after arthroscopic shoulder surgery. Acta Anaesthesiol Scand. 2002;46(8):1031-6.

13. Muittari PA, Nelimarkka O, Seppälä T, Kanto JH, Kirvelä OA. Comparison of the analgesic effects of intrabursal oxycodone and bupivacaine after acromiplasty. J Clin Anesth. 1999;11(1):11-6.

14. Almeida A, Roveda G, Valin MR, Valin MCS, Almeida NC, Agostini AP. Analgesia para a sutura artroscópica do manguito rotador: estudo comparativo entre o bloqueio interescalênico do plexo braquial e o bloqueio da bursa subacromial contínuo. Rev Bras Ortop. 2007;42(10)324-32.

15. Singelyn FJ, Lhotel L, Fabre B. Pain relief after arthroscopic shoulder surgery: a comparison of intra-articular analgesia, suprascapular nerve block, and interscalene brachial plexus block. Anesth Analg. 2004;99(2):589-92. 